\title{
Methodological challenges in carbohydrate analyses
}

\author{
Mary Beth Hall
}

U. S. Dairy Forage Research Center, USDA-ARS, Madison, WI 53561.

\begin{abstract}
Carbohydrates can provide up to $80 \%$ of the dry matter in animal diets, yet their specific evaluation for research and diet formulation is only now becoming a focus in the animal sciences. Partitioning of dietary carbohydrates for nutritional purposes should reflect differences in digestion and fermentation characteristics and effects on animal performance. Key challenges to designating nutritionally important carbohydrate fractions include classifying the carbohydrates in terms of nutritional characteristics, and selecting analytical methods that describe the desired fraction. The relative lack of information on digestion characteristics of various carbohydrates and their interactions with other fractions in diets means that fractions will not soon be perfectly established. Developing a system of carbohydrate analysis that could be used across animal species could enhance the utility of analyses and amount of data we can obtain on dietary effects of carbohydrates. Based on quantities present in diets and apparent effects on animal performance, some nutritionally important classes of carbohydrates that may be valuable to measure include sugars, starch, fructans, insoluble fiber, and soluble fiber. Essential to selection of methods for these fractions is agreement on precisely what carbohydrates should be included in each. Each of these fractions has analyses that could potentially be used to measure them, but most of the available methods have weaknesses that must be evaluated to see if they are fatal and the assay is unusable, or if the assay still may be made workable. Factors we must consider as we seek to analyze carbohydrates to describe diets: Does the assay accurately measure the desired fraction? Is the assay for research, regulatory, or field use (affects considerations of acceptable costs and throughput)? What are acceptable accuracy and variability of measures? Is the assay robust (enhances accuracy of values)? For some carbohydrates, we may have to accept that there are currently no acceptable, widely applicable analyses that meet these criteria, and seek to fill this analytical gap. Overall, there are good possibilities for accomplishing the nutritional partitioning of carbohydrates. But to ensure that this is done well, as a discipline, we need to continue our discussions on the basis for partitioning, on what fractions are actually significant, and rigorously evaluate proposed analyses to verify that they are analytically sound.
\end{abstract}

Key words: analysis, carbohydrate, fiber, starch, sugars

\section{Introduction}

Carbohydrates comprise up to $80 \%$ of the dry matter in ruminant diets, and represent a substantial proportion of the diets of many other species. Although dietary carbohydrates are diverse in their compositional and digestion characteristics (Figure 1), there has been little specific analysis of them for use in diet formulation. As early as 1890 , it was recommended that carbohydrate analyses be improved to better estimate their nutritive value (Wiley, 1890). As we have progressed from cows making $6,800 \mathrm{~kg}$ of milk per year to $13,600 \mathrm{~kg}$, and from diets in which forage predominates to diets with greater amounts of concentrates that carry increased risk of causing digestive upset
(Sutton et al., 1987), the need for predicting the dietary effects of carbohydrates become apparent. A more detailed understanding of and methods of measuring dietary carbohydrates that have potential to affect animal performance are needed if we are to objectively use carbohydrates to formulate diets that support desired animal production and health.

\section{Method selection considerations}

The first step in method selection is deciding what to measure. For use in diet formulation, carbohydrate partitioning should reflect differences among carbohydrates in digestion and fermentation characteristics, yield of nutrients, and

Correspondências devem ser enviadas para: MaryBeth.Hall@ars.usda.gov USDFRC, 1925 Linden Drive, Madison, WI 53706, USA. Phone: 608-890-0078 


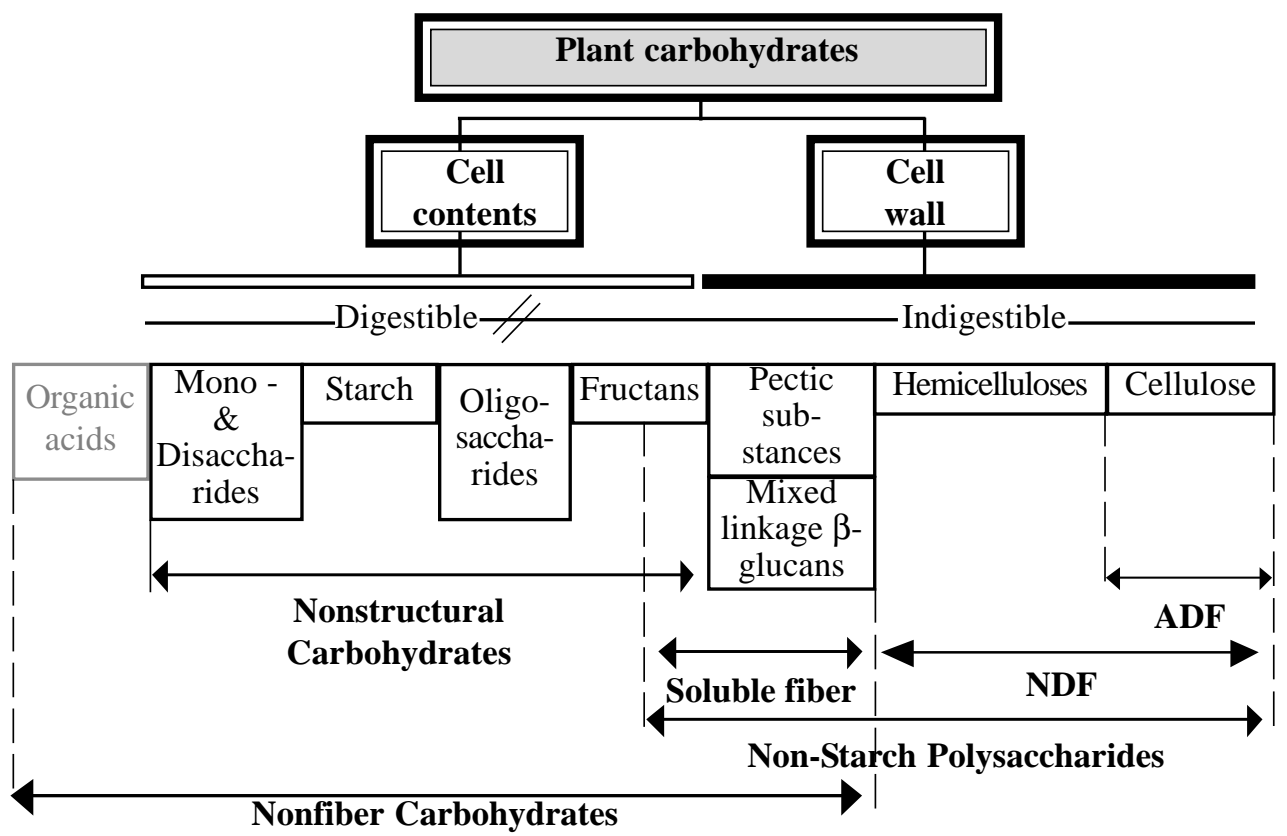

Figure 1 - Carbohydrates in plants. Digestible or Indigestible refer to potential for digestion by enzymes in the small intestine; all carbohydrates shown are potentially fermentable. Organic acids are not a carbohydrate, but their mass is included in the nonfiber carbohydrate value that is calculated by difference. As shown, soluble fiber includes only non-starch polysaccharides not in NDF. NDF = neutral detergent fiber, $\mathrm{ADF}=$ acid detergent fiber.

effects on animal performance. The challenge is deciding which fractions are significant in their effect and dietary content to be measured, but not have so many fractions that the system becomes unworkable. The lack of information on digestion characteristics of some carbohydrates and their interactions in diets means that the partitioning will not soon be perfectly established.

Once the nutritionally important carbohydrate fractions are defined, selection of analytical methods begins. Any method considered must measure the desired analytes. Choice of method requires the setting of acceptable levels of accuracy and allowable variability of measurements. These will be affected by the robustness of the assay, and the effects of factors/substances that can inflate or decrease results. The assays should be evaluated on the variety of feedstuffs that will be analyzed, and on purified substrates to test the specificity of the assay. Robustness of the methods should also be considered - all things equal, the more robust the better to ensure consistent, reliable results. Another consideration is cost and availability of the analyses. Some methods are available that can very specifically measure the analytes of interest but at significant costs of time and money using expensive pieces of equipment that by virtue of ๑ 2007 Sociedade Brasileira de Zootecnia cost may limit the number of samples that can be analyzed. Alternative methods may be less precise or more variable, but, if the errors are not excessive, can be utilized by more laboratories at lesser costs. Which method is acceptable for use will depend on the use of the results (research, feed industry regulation, diet formulation). Concerns beyond the accuracy of the method do not justify shoddy science or analyses, but, on a practical basis, they bear consideration. With our present state of the art, analyses that meet criteria for specificity, cost, and availability may or may not exist for a given carbohydrate fraction.

The carbohydrate methods currently available include both empirical and non-empirical analyses. Non-empirical analyses measure specific analytes (e.g., glucose, sucrose, starch), whereas in empirical analyses the method used defines what is measured (e.g., neutral detergent fiber, watersoluble carbohydrates, Klason lignin). The empirical methods can be quite useful, but have potential for the actual composition of the material they measure to vary by feedstuff. With empirical methods, it is absolutely essential that the established method be followed precisely or the results will not describe the intended measure. 
Caution is urged regarding choosing methods that were developed for use outside of the field of nutrition. The methods may or may not be useful for nutritional purposes. For instance, the study of carbohydrates in plants has long been the realm of agronomists. As they sought to understand how plants allocated products of photosynthesis, they performed detailed analyses of the carbohydrate fractions in crops (e.g., Ojima \& Isawa, 1968). The agronomic analyses of nonstructural carbohydrate (monosaccharides, oligosaccharides, fructans, starch) and cell wall carbohydrate (cellulose, hemicelluloses, pectins, mixed linkage betaglucans) describe groupings of carbohydrates that are or are not readily mobilized by the plant (Smith, 1981). The nonstructural carbohydrates in particular contain diverse carbohydrates that are heterogeneous in their digestion and fermentation characteristics; this fraction is not recommended for use in nutritional applications.

\section{Carbohydrate classification}

Is it essential to have different systems of analysis for simple stomached and ruminant animals? All animals rely to one extent or another on digestion of feedstuffs with enzymes produced by the animal and by microbial fermentation. Carbohydrate fractions also need to be divided by digestibility by endogenous enzymes, fermentation characteristics, and possibly physical effects. A system of carbohydrate partitioning that could be applied across animal species would have the advantages of establishing a coherent set of analyses useful in more disciplines and of allowing development of a greater body of research information describing animal responses to dietary carbohydrates. It would be necessary to use the fractions differently in diet formulation among species. If there are carbohydrates peculiar to diets fed to certain species that have substantial impact on animal performance, specific analyses of those carbohydrates would be warranted.

The Food and Agriculture Organization of the United Nations (FAO)(1998) suggested a scheme for classification of carbohydrates for use in human nutrition that, with some modification, may serve the animal science community. The 3 categories with subcategories of the FAO list are sugars (degree of polymerization (DP) $=1$ to 2 ) which include monosaccharides, disaccharides, and sugar alcohols (Note: the plural sugars denotes monoand disaccharides, whereas the singular sugar refers to sucrose or table sugar), oligosaccharides (DP $=3$ to 9 ) which are separated into maltooligosaccharides and other oligosaccharides (stachyose, raffinose, fructo-oligosaccharides), and polysaccharides which are separated into starch and nonstarch polysaccharides. Note that within categories, the list explicitly separates carbohydrates that are digestible by mammalian enzymes (malto-oligosaccharides, starch) from those that are not (other oligosaccharides, nonstarch polysaccharides). Assays are not currently available to readily analyze all subcategories of carbohydrates (D.R. Lineback, personal communication). Based on the research literature, the fractions that seem most relevant to diet characterization for the animal science community are sugars, starch, fructans, insoluble fiber, and soluble fiber.

\section{Sugars}

Many analyses have been used to make estimates of the content of sugars in feedstuffs, but it is unlikely that most of them meet the definition of specifically measuring monosaccharides plus disaccharides. Extraction of samples with $80 \%$ ethanol extracts sugars as well as larger oligosaccharides, though polysaccharides should remain largely unextracted (Asp, 1993); analysis of such extracts will not give a completely reliable value for sugars. The degree to which oligosacchrides inflate the value will vary with the oligosaccharide content of the feedstuffs and the method of carbohydrate detection. Analysis of the extract with the phenol - sulfuric acid assay (Du Bois et al., 1956), a broad spectrum analysis that can detect carbohydrate polymers and monomers, will detect all carbohydrates in the extract, but the values will vary with the carbohydrate used as the standard for the assay. Reducing sugar assays cannot be performed on extracts containing ethanol (Southgate, 1976).

Water extracts the low molecular weight carbohydrates that ethanolic extractions also remove, but can also extract water-soluble polysaccharides. If analyzed with the phenol sulfuric acid assay, all of these carbohydrates will 
be detected, making this an unreliable measure of sugars. If a reducing sugar analysis is used only the monosaccharides will be detected, unless the disaccharides in the extract are hydrolyzed, and total of native and released monosaccharides measured. Acid hydrolysis procedures designed to hydrolyze sucrose (which hydrolyzes more rapidly other disaccharides) can also hydrolyze oligosaccharides such as stachyose, raffinose, and fructooligosaccharides that contain fructofuranosides (Pomeranz \& Meloan, 1987). Use of an array of enzymes (sucrase, lactase, maltase) could be used to attempt specific hydrolysis of the disaccharides, but care should be taken that the activity of the enzyme preparations does not extensively hydrolyze other carbohydrates (e.g., invertase can hydrolyze raffinose; Pomeranz \& Meloan, 1987, and fructans, Megazyme, 2004). As regards additional sources of interference in the colorimetric measurement of sugars, reducing sugar analyses can be subject to interference from protein and from reducing substances present in the sample (Southgate, 1976). However, it has been reported that some reducing sugar analyses are less subject to interference (Lever, 1972) than others.

It would appear that a complete accounting of mono- and disaccharides with standard chemical methods may be a challenge. This author believes that investigation of a sugar method that uses specific enzymatic hydrolysis of disaccharides accompanied by total measurement of monosaccharides should be evaluated as a method of analysis for sugars — it could be widely used using common laboratory equipment. Such a method would not differentiate among the specific sugars present, but would provide a total value for this fraction.

Chromatographic methods generally offer the greatest specificity for measurement of sugars and provide the gold standards for low molecular weight carbohydrate analysis. Both high performance liquid chromatography (Bach Knudsen, 1997) and high performance anion exchange chromatography with pulsed amperometric detection (Kennedy \& Pagliuca, 1994) can offer great specificity, sensitivity, and freedom from interferences. The equipment is expensive, sample throughput may be decreased as compared to the colorimetric assays, and sample

๑ 2007 Sociedade Brasileira de Zootecnia preparation may be relatively minimal to extensive, depending upon the potential contaminants in the samples. Gas chromatography with derivitization of monosaccharides to alditol acetates does not allow for resolution of some pairs of sugars (e.g., glucose/gulose) because they are reduced to the same alditol, and does not allow definitive analysis of fructose: two different isomeric alditol acetates are produced from a ketose as compared to one for aldoses such as glucose (Chaplin, 1994).

Generally, the recommendation for sugar or starch analysis is to grind samples through a 0.5 mm screen $(1.0 \mathrm{~mm}$ screen on an abrasion mill (e.g., Udy or cyclone) is equivalent).

\section{Starch}

Starch encompasses the $\alpha$-glucan polysaccharides. These include amylose, amylopectin, and glycogen all of which contain $\alpha-(1->4)$ and $\alpha-(1->6)$ linkages. Native starch is present as cold water-insoluble granules with a crystalline structure from the hydrogen bonding among the amylose and amylopectin molecules. In grains, the granules are embedded in a protein matrix. Disruption of the protein matrix around the granules through fine grinding may be essential to allow solubilization/hydrolysis of the starch.

The two most common methods of starch analysis are polarimetry and enzymatic analysis. Polarimetry is commonly used on cereal grains and may be subject to interference from sugars, fructans, and carbonates among other feed components (CONSLEG, 1999). Enzymatic analyses rely on the specificity of enzymes to separate starch from other glucose containing carbohydrates. Critical elements for accurate enzymatic analysis of starch are complete gelatinization of starch, specificity of enzymes, complete hydrolysis of starch to glucose, specific measurement of glucose released from starch hydrolysis, and minimization of interference.

Gelatinization involves the dissolution of hydrogen bonds among and within starch molecules to open the molecules up to hydration and enzymatic hydrolysis. The crystalline structure of the granules must be disrupted for complete enzymatic hydrolysis of the starch to take place in a reasonable amount of time. Gelatinization is 
typically accomplished with heating $\left(90\right.$ to $\left.100^{\circ} \mathrm{C}\right)$ with water or buffer, or, alternatively, with use of a base (e.g., potassium hydroxide) followed by neutralization (Englyst et al., 1982). Incomplete gelatinization can lead to incomplete hydrolysis of starch to glucose.

Enzymatic hydrolysis of only the $\alpha-(1->4)$ (linear chain) and $\alpha$-(1->6) (branches) linkages present in starch is the element that makes a method specific for starch and $\alpha$-glucans. Select enzyme preparations that do not contain the carbohydrate to be measured (e.g., amylases for dietary fiber determinations may contain glucose). Thermostable $\alpha$-amylase (an endoamylase), which can be added during the gelatinization step, and amyloglucosidase (an exoamylase), which hydrolyzes $\alpha$-glucan to glucose from the nonreducing ends of the molecules, are commonly used. Enzymes must be incubated at the correct pHs and temperatures to optimize their ability to hydrolyze $\alpha$-glucan, otherwise the hydrolysis may not go to completion and starch content will be underestimated. In the past, the optimal $\mathrm{pH}$ for the thermostable $\alpha$-amylases used to be close to neutrality, but newer amylases can function at lower $\mathrm{pH}$ (5.0). Amyloglucosidase has optimal activity at 4.5 to 5.0 . The more acid-tolerant amylases have the advantage of allowing the entire assay to be performed in $\mathrm{pH} 5.0$ sodium acetate buffer (Bach Knudsen, 1997), thus reducing the number of sample transfers. Even more importantly, running the assay at $\mathrm{pH} 5.0$ greatly reduces the production of maltulose, a glucosefructose disaccharide, that reduces starch recovery. Maltose is produced at neutral $\mathrm{pH}$ and high temperature when the reducing end glucose on a starch molecule isomerizes to fructose (Dias \& Panchal, 1987). During gelatinization and liquefaction of starch with heat and thermostable amylase at neutral $\mathrm{pH}$, likelihood of isomerization increases as the number of reducing ends produced by enzymatic hydrolysis increases. Amyloglucosidase hydrolyzes the starch molecule up to this disaccharide, but cannot hydrolyze the glucose - fructose bond. Dias \& Panchal (1987) reported that maltulose formation during saccharification of starch was dependent on $\mathrm{pH}$ and temperature. They reported that maltulose accounted for up to 5 to $6 \%$ of total sugars at $\mathrm{pH}$ 6.5 to 7.0 , which reduced the yield of glucose from starch commensurately. Decreasing the $\mathrm{pH}$ to between 4.5 and 5.5 at $121^{\circ} \mathrm{C}$ reduced maltulose formation to approximately $1 \%$ of total sugars.

Measurement of glucose released from starch hydrolysis should be accomplished with an analysis specific for glucose, such as a glucose oxidase-peroxidase assay (e.g., Karkalas, 1985). Reducing sugar analysis is not recommended for detection of released glucose, as it also will detect all other monosaccharides released/present. Starch content of the feed is calculated as glucose content times 0.9. This calculation accounts for the removal of one molecule of water for each molecule of glucose during the covalent bonding of glucose molecules to form starch. High purity $(\geq 99.5 \%)$ glucose should be used as the standard for the end product assay. Use of starch as the standard is not recommended because it relies upon complete recovery (starch measured/actual starch), and presumes similar recoveries for starch from all sources. Use of glucose as the standard removes the question of percent recovery. The author strongly recommends using glucose and purified starch samples as control samples during starch assays. They allow assessment of completeness of glucose recovery independent of and with assessment of enzyme, assay conditions, and technical efficacy.

To avoid inflation of starch values, free glucose present in the sample and glucose released from non-starch carbohydrates need to be evaluated. Free glucose may be analyzed on sample extracts prepared by running them through the assay but with no enzymes. This value for free glucose should be subtracted from the value for enzymatically released glucose. Release of glucose from non-starch carbohydrates can be tested in part by applying the starch analysis to suspect carbohydrates -- appreciable released glucose should not be detected. If significant amounts of glucose are detected, evaluation of enzymes and run conditions are in order. Sucrose is a prime candidate for hydrolysis due to run conditions (addition of acid to stop enzyme activity in some assays) or side activity of the enzymes (e.g., invertase) (Hall et al., 2000). If side activity of the enzymes is the issue, and alternative enzyme sources cannot be found, preextraction of samples with $80 \%$ ethanol will remove the low molecular weight carbohydrates that are a likely source of the interference.

() 2007 Sociedade Brasileira de Zootecnia 
There has been discussion on the use of corrections to adjust sample starch values for the recovery of a starch control sample. The hazards with this approach are that it presumes that starch in all samples behaved similarly to the control, and that the low or high recoveries are not a function of unacceptable problems with the technique, enzymes, etc. Generally speaking, the author would recommend against such corrections.

There are differing opinions in the field of human nutrition as to whether maltose and maltooligosaccharides should be separate from or included with starch. Their separation from starch could be accomplished with an $80 \%$ ethanol preextraction of samples. We need to evaluate whether these carbohydrates have sufficient nutritional impact and are in sufficient quantities in feedstuffs to be concerned about them for method selection, modification of assays, and sample handling.

Resistant starch is resistant to digestion by small intestinal enzymes (Englyst et al., 1982) and has been considered to be a form of dietary fiber. In the author's opinion, it is far preferable to determine the total starch content of a feedstuff, and then apply descriptors to describe the digestion characteristics of the starch (resistant, digestible, having differing rates of fermentation). Consider that digestibility of starch has potential to differ across animal species as it is subject to differing extents of fermentation and enzymatic digestion, different descriptors will likely be needed by specie, and perhaps by other animal characteristics (maturity, level of dry matter intake, etc.).

\section{Fructans}

Fructans include compounds where one or more fructosyl- fructose linkages constitute a majority of the linkages (Lewis, 1993). Unlike other carbohydrates, the term fructan is inclusive of fructo-oligosaccharides and polysaccharides. These carbohydrates are commonly found in temperate, cool season grasses, but are also found in chicory (Cichorium intybus), Jerusalem artichoke, and onions. They are indigestible to mammalian enzymes (Nilsson et al., 1988), but may be fermented to produce lactic acid (Muller $\&$ Steller, 1995). The lack of digestion in the small intestine combined with fermentation characteristics reminiscent of sugars and starch set them apart from other carbohydrates. Their fermentation characteristics have not been well described (Dewhust et al., 2000). There have been indications that fructans may have potential negative effects on animal health (Thoefner et al., 2004).

Fructans can be extracted with water and analyses performed on the extract. An enzymatic fructan assay has been developed for foods using an enzyme that is effective on chicory fructan (inulin; McCleary et al., 1997), but evaluation of the effectiveness of the enzymes on non-chicory fructans is recommended. Extracted fructans can be gently hydrolyzed with acid to release monosaccharides which are then measured (Bach Knudsen, 1997). In either procedure, sucrose and monosaccharides must be accounted for so they do not inflate the fructan value.

\section{Neutral detergent fiber (NDF)}

Neutral detergent fiber is equivalent to insoluble dietary fiber in the human nutrition system of food analysis (Mongeau \& Brassard, 1986). In ruminant nutrition in particular, this empirical assay has become established as a measure of dietary fiber that can be related to dietary fractions that are typically but not always more slowly fermented. It has also been combined with assessment of physical form of feeds in the diet as an indicator of the adequacy of the diet to maintain rumen function (Mertens, 1997).

There have been many modifications made to the NDF method, but as an empirical method, if the method is not followed precisely according to the established protocol, the analyte measured is not NDF. Alternative NDF procedures should be compared to the standard to verify the accuracy of their measurements. The NDF procedure using sodium sulfite to remove protein and thermostable $\alpha$-amylase to remove starch has received AOAC approval (Mertens, 2002). Techniques such as using sand as a filtration aid and requiring soaking rather than rinsing of the residue to remove residual detergent have improved the accuracy of the assay. The NDF method was designed for forages, and may not be appropriate for all types of animal feeds (e.g., some forms of wild mushrooms become slimy and will not filter with this procedure; Hanson et al., 2006). That would

\footnotetext{
๑ 2007 Sociedade Brasileira de Zootecnia
} 
recommend that another dietary fiber method be explored for those samples.

In terms of nutritional relevance, NDF has served the animal science community far better than crude fiber. As with the discussions in the human nutrition community, it would serve the animal science community well to keep an open mind, and continue to explore how well NDF and other methods serve its nutritional needs and what changes in methods would better serve us.

\section{Soluble fiber}

Before someone gets upset at the notion that fiber can be soluble, remember that nutritionally, fiber can be defined as the nondigestible carbohydrates and lignin that are intrinsic and intact in plants (dietary fiber) (Institute of Medicine, 2001). Claims of solubility raise the question: Soluble in what solvent and under what conditions? The soluble fiber referred to here includes the nonstarch polysaccharides that are soluble in neutral detergent or buffers, and so are not a part of NDF or insoluble dietary fiber. They include pectic substances, mixed linkage $\beta$ glucans, the longer chain length fructans, and other carbohydrates of similar solubility and digestibility. This is a compositionally diverse fraction, and further work is needed to determine the acceptability of grouping these carbohydrates together.

The current approved methods for soluble fiber have challenges. The Association of Official Analytical Chemists (AOAC) soluble dietary fiber methods (Prosky et al., 1992) solubilize the carbohydrates in phosphate (AOAC method 985.29) or MES-TRIS (AOAC method 991.43) buffers, and remove starch and protein by treatment with amyloglucosidase and protease, respectively. After filtration to separate it from insoluble dietary fiber, solubilized dietary fiber is precipitated from the buffer extract with $78 \%$ ethanol and is determined gravimetrically with correction for protein and ash. This method does not include short chain fructans in the soluble fiber fraction, nor long chain if inulinase is used. Reliance on ethanol precipitation for recovery may result in incomplete precipitation of soluble fiber and coprecipitation of other organic compounds (Mañas \& Saura-Calixto, 1993). The recovery of soluble dietary fiber after precipitation with $78 \%$ ethanol or dialysis $(12,000$ to 14,000 molecular weight cutoff) were $5.34 \%$ and $12.33 \%$ of dry matter, respectively for orange peel. The magnitude of difference between the two recovery methods differed by foodstuff (Mañas \& SauraCalixto, 1993).

An alternative measure of soluble fiber is that for neutral detergent-soluble fiber (nonstarch, nonNDF polysaccharides soluble in neutral detergent plus heat-stable, $\alpha$-amylase). The polysaccharides found in starch, soluble fiber, and insoluble fiber comprise the carbohydrate constituents of the residue remaining after extraction with $80 \%$ ethanol (Theander \&Westerlund, 1986). Rather than attempting precipitation of soluble fiber after its extraction, soluble fiber may be determined gravimetrically as the difference between the weight of the $80 \%$ ethanol-insoluble residue and those of starch and insoluble fiber (NDF), with corrections for protein and ash (Hall et al., 1999). This system of analysis requires concurrent determination of starch and insoluble fiber. Only the larger fructans that are insoluble in $80 \%$ ethanol will be counted as soluble fiber by this assay. Although the constituent assays have good repeatability and are simpler than the soluble dietary fiber method, estimation of soluble fiber by difference leaves the potential for errors from the component assays to accumulate in the estimate. The errors inherent in correcting for protein as $\mathrm{N} x 6.25$, with the incorrect presumption that 6.25 is the correct multiplier for all samples (Jones, 1931), affects both soluble fiber methods.

There is much debate in the human nutrition community as to whether soluble fiber and insoluble dietary fiber should be measured separately, considering that their individual physiological effects are not necessarily consistent (Food and Agriculture Organization, 1998). In animal nutrition, if NDF is used as a measure of insoluble fiber, some accounting of soluble fiber may be recommended to more fully account for carbohydrates in the diet, because neither the NDF analysis nor the other methods mentioned include them. Soluble fiber may make up from 5 to $10 \%$ of diet dry matter (Hall and Van Horn, 2001), possibly more if much legume forage or byproduct feeds such as citrus pulp or sugar beet pulp are fed.

두 2007 Sociedade Brasileira de Zootecnia 
Summary

In this paper, I have presented a small portion of the picture of the state of carbohydrate analysis for animal nutrition. There are good possibilities for accomplishing the nutritional partitioning of carbohydrates. But to ensure that this is done well, as a discipline, we need to continue our discussions on the basis for partitioning, on what fractions are actually significant, and rigorously evaluate proposed analyses to verify that they are analytically sound.

\section{References}

ASP, N.G. Nutritional importance and classification of food carbohydrates. In: MEUSER, F.; MANNERS, D.J.; SEIBEL, W. (Ed.) Plant polymeric carbohydrates. Cambridge: Royal Society of Chemistry, 1993. p.121-126.

BACH KNUDSEN, K.E. Carbohydrate and lignin contents of plant materials used in animal feeding. Animal Feed Science and Technology, v.67, p.319-338, 1997.

CHAPLIN, M.F. Monosaccharides. In: CHAPLIN, M.F.; KENNEDY, J.F. (Ed.) Carbohydrate analysis-a practical approach. 2.ed.. New York: Oxford University Press, Inc., 1994. p.1-41.

CONSLEG. Third Commission Directive of 27 April 1972 establishing Community methods of analysis for the official control of feedingstuffs. Amended by M5 Commission Directive 1999/79/EC of 27 July 1999 L209. Office for Official Publications of the European Communities, 1999. p.4-6.

DEWHURST, R.J.; DAVIES, D.R.; MERRY, R.J. Microbial protein supply from the rumen. Animal Feed Science and Technology, v.85, p.1-21, 2000.

DIAS, F.F.; PANCHAL, D.C. Maltulose formation during saccharification of starch. starch/stärke, v.39, n.2, S, p.6466,1987

DUBOIS, M.; GILLES, K.A.; HAMILTON, J.K. et al. Colorimetric method for determination of sugars and related substances. Analytical Chemistry, v.28, p.350-356, 1956.

ENGLYST, H.; WIGGINS, H.S.; CUMMINGS, J.H. Determination of the nonstarch polysaccharides in plant foods by gas-liquid chromatography of constituent sugars as alditol acetates. Analyst, v.107, p.307-318, 1982.

FOOD AND AGRICULTURE ORGANIZATION Carbohydrates in human nutrition. Food and Agriculture Organization of the United Nations, Rome, Italy. 1997. p.113 (FAO Food and Nutrition Paper - 66).

HALL, M.B.; HOOVER, W.H.; JENNINGS, J.P.et al. A method for partitioning neutral detergent-soluble carbohydrates. Journal of the Science of Food and Agriculture, v.79, p.2079-2086, 1999.

HALL, M.B.; JENNINGS, J.P.; LEWIS, B.A. et al. Evaluation of starch analysis methods for feed samples. Journal of the Science of Food and Agriculture, v.81, p.17-21, 2000

HALL, M.B.; VAN HORN, H.H. How should we formulate for non-NDF carbohydrates? IN: PROCEEDINGS OF THE $12^{\text {TH }}$ ANNUAL FLORIDA RUMINANT NUTRITION SYMPOSIUM, Gainesville, Florida. University of Florida Department of Animal Sciences, 2001, p.44.

HANSON, A.M.; HALL, M.B.; PORTER, L.M. et al. Composition and nutritional characteristics of fungi consumed by Callimico goeldii in Pando, Bolivia.
International Journal of Primatology, 2006. Online publication at http://dx.doi.org/10.1007/s10764-005-9014-z.

INSTITUTE OF MEDICINE. Dietary reference intakes: proposed definition of dietary fiber. Washington, DC:National Academy Press, 2001. 74p.

JONES, D.B. Factors for converting percentages of nitrogen in foods and feeds into percentages of proteins. United States Department of Agriculture, 1931. p.1-21 (Circular No. 183).

KARKALAS, J.J. An improved enzymatic method for the determination of native and modified starch. Journal of the Science of Food and Agriculture, v.36, p.1019-1027, 1985.

KENNEDY, J.F.; PAGLIUCA, G. Chapter 2. Oligosaccharides. In: CHAPLING, M.F.; KENNEDY, J.F. (Ed.) Carbohydrate analysis-a practical approach. 2.ed.. New York: Oxford University Press, Inc., 1994. p.43-72.

LEVER, M. A new reaction for colorimetric determination of carbohydrates. Analytical Biochemistry, v.47, p.273-279, 1972.

LEWIS, D.H. Nomenclature and diagrammatic representation of oligmeric fructans - a paper for discussion. New Phytologist, v.124, p.583-594, 1993

MAÑAS, E.; SAURA-CALIXTO, F. Ethanolic precipitation: a source of error in dietary fibre determination. Food Chemistry, v.47, p.351-355, 1993

MCCLEARY, B.V; MURPHY, A.; MUGFORD, D.C. Determination of oligofructans and fructan polysaccharides in foodstuffs by an enzymatic/spectrophotometric method: collaborative study. Journal of the Association of Official Analytical Chemists International, v.83, p.356-364, 1997.

MEGAZYME. Fructan assay procedure for the analysis of fructo-oligosaccharides (FOS) and fructan polysaccharide (data booklet). Megazyme International Ireland Limited, Bray, Ireland., 2004. 11p.

MERTENS, D.R. Creating a system for meeting the fiber requirements of dairy cows. Journal of Dairy Science, v.80, n.7, p.1463-1481, 1997.

MERTENS, D.R. Gravimetric determination of amylase-treated neutral detergent fiber in feeds with refluxing in beakers or crucibles: collaborative study. Journal of the Association of Official Analytical Chemists, v.85, p.1217-1240, 2002.

MONGEAU, R.; BRASSARD, R. A rapid method for the determination of soluble and insoluble dietary fiber: comparison with AOAC total dietary fiber procedure and Englyst's method. Journal of Food Science, v.51, p.13331336, 1986

MULLER, M.; STELLER, J. Comparative studies of the degradation of grass fructan and inulin by strains of Lactobacillus paracasei subsp. paracasei and Lactobacillus plantarum. Journal of Applied Bacteriology, v.78, p.229-236, 1995.

NILSSON, U.; OSTE, R.; JAGERSTAD, M. et al. Cereal fructans: in vitro and in vivo studies on availability in rats and humans. Journal of Nutrition, v.118, p.1325-1330, 1988.

OJIMA, K.; ISAWA, T. The variation of carbohydrates in various species of grasses and legumes. Canadian Journal of Botany, v.46, p.1507-1511, 1968.

POMERANZ, Y.; MELOAN, C.E. Food analysis: theory and practice. 2.ed. New York: Van Nostrand Reinhold, 1987. 797p.

PROSKY, L.; ASP, N.G.; SCHWEIZER, T.F. et al. Determination of insoluble and soluble dietary fiber in foods and food products: collaborative study. Journal of the Association of Official Analytical Chemists, v.75, p.360367, 1992.

SMITH, D. Removing and analyzing total nonstructural carbohydrates from plant tissue. Research Division, College of Agriculture and Life Sciences, University of Wisconsin, Madison, 1981. p.1-13 (Research report R2107). SOUTHGATE, D.A.T. Determination of food carbohydrates. London:Applied Science Publishers, Ltd, 1976. 178p.

๑) 2007 Sociedade Brasileira de Zootecnia 
SUTTON, J.D.; BINES, J.A.; MORANT, S.V. et al. A comparison of starchy and fibrous concentrates for milk production, energy utilization and hay intake by Friesian cows. Journal of Agricultural Science (Cambridge), v.109, p.375-386, 1987.

THEANDER, O.; WESTERLUND, E.A. Studies on dietary fiber. 3. Improved procedures for analysis of dietary fiber. Journal of Agriculture and Food Chemistry, v.34, p.330336, 1986.

THOEFNER, M.B; POLLITT, C.C.; VAN EPS, A.W. et al. Acute bovine laminitis: a new induction model using alimentary oligofructose overload. Journal of Dairy Science, v.87, p.2932-2940, 2004.

WILEY, H.W. Report of the committee on ways and means for securing more thorough chemical study of foods and feeding stuffs, Proceedings of the seventh annual convention of the Association of Official Agricultural Chemists. U.S. Department of Agriculture, Division of Chemistry, 1890, p.117-125 (Bulletin No.28). 